\title{
Producción científica sobre integración de TIC a la Educación Física. Estudio bibliométrico en el periodo 1995-2017 \\ Scientific production on the integration of ICT to Physical Education. Bibliometric study in the period 1995-2017 \\ Juan Francisco Cabrera Ramos \\ Universidad Católica de Temuco (Chile)
}

Resumen. En las dos últimas décadas, la literatura ha aportado argumentos de forma sistemática sobre la utilidad de la integración de las Tecnologías de la Información y la Comunicación (TIC) en la Educación Física. El objetivo de la presente investigación es describir el comportamiento de la producción científica en el área de las TIC y la Educación Física en el período de 1995 a 2017. El estudio se basa en el análisis bibliométrico de publicaciones recopiladas en diversos repositorios de publicaciones científicas. Se recolectó un total de n=521 contribuciones, que incluyen artículos científicos, presentaciones en eventos científicos, tesis, libros y capítulos de libros. Se determinan los autores más productivos, las principales fuentes de información, las principales líneas de investigación y las contribuciones más influyentes. Se encuentra un alto grado de colaboración nacional, un bajo nivel de colaboración internacional y un limitado número de autores con más de una publicación en el tema. En el análisis sobre la madurez del dominio se considera que estamos ante un dominio de conocimientos en fase de crecimiento.

Palabras clave: Pedagogía, Educación Física, Integración de las TIC, Bibliometría, Colaboración.

Abstract. In recent decades, scientific literature has provided arguments about the integration of Information and Communication Technologies (ICT) in Physical Education in a systematic way. The objective of this research is to describe the trend of scientific production in the area of ICT and Physical Education in the period from 1995 to 2017. The study is based on the bibliometric analysis of publications collected in various databases of scientific publications. A total of $n=521$ contributions were collected, including scientific articles, presentations at scientific events, theses, books, and book chapters. The most productive authors, the main sources of information, the main areas of research, and the most influential contributions were determined. There is a high degree of national collaborations but a low level of international ones, and a low number of authors with more than one publication on the subject. In the domain maturity analysis, we can consider this topic as in a growth phase.

Key Words: Pedagogy, Physical Education, ICT Integration, Bibliometrics, Collaboration.

\section{Introducción}

El avance de las Tecnologías de la Información y la Comunicación (TIC), la masificación del acceso y su disponibilidad en el sistema escolar, así como el incremento de acciones orientadas a la capacitación de docentes en el área de las TIC, configuran hoy un panorama bastante estable en América Latina.

Aun cuando se reconoce la utilidad de las TIC en todas las materias del sistema educativo y varios autores hacen patente su potencial y la urgencia de que se reconozcan como un medio clave dentro del proceso de formación en la Educación Física (Araújo, Batista, \& Moura, 2017; Campos \& Castillo, 2016; Macdonald, 2015; Papastergiou, 2009; Quijada, 2015), en la literatura aparecen frecuentes referencias a declaraciones de docentes sobre la irrelevancia y la complejidad de la integración de TIC en la Educación Física (Baert, 2011a; Bauer \& Kenton, 2005; Kretschmann, 2015), donde se plantea que restan tiempo de actividad física, distraen, afectan la socialización, entre otros tantos argumentos.

En discusiones sostenidas con docentes sobre la necesidad de integrar las TIC en la Educación Física en el contexto regional latinoamericano, además de los planteamientos anteriores, ha sido recurrente la idea de que se ha escrito muy poco sobre el tema y que está asociado sólo a países con alto nivel de desarrollo económico. Aun cuando no se comulga con la idea de que se ha publicado poco sobre el

Fecha recepción: 04-09-18. Fecha de aceptación: 11-07-19 Juan Francisco Cabrera Ramos

jcabrera@uct.cl tema, sí se ha podido constatar, en una primera revisión, que existe muy poca sistematización de la producción intelectual en el tema.

En este contexto se considera relevante que se explore lo publicado en el tema a través del tiempo: quiénes y en qué contexto han hecho las contribuciones al área de conocimiento, qué estructuras de colaboración han seguido, en qué fuentes se ha publicado las principales contribuciones, cuán madura es el área de conocimientos, cuánto se cita la producción en el área. Estas, entre otras interrogantes permiten establecer una mirada holística sobre cuánto se ha avanzado en el dominio de conocimientos.

En la revisión de la literatura científica se identifican barreras que dificultan este ejercicio integrador: falta de coherencia sobre metadatos utilizados en la clasificación de contribuciones, diversidad de fuentes en que se han publicado contribuciones y la pluralidad de índices en que se pueden encontrar contribuciones sobre el tema, pero en su mayoría con resultados parciales. La mayoría de los índices disponibles consideran exclusivamente las bases de datos de alto impacto e ignoran contribuciones que, con una cualidad equivalente, fueron publicadas en revistas con menor grado de reconocimiento o en momentos donde la publicación en revistas de alto impacto no era considerado relevante. Es por ello que se establece la necesidad de llevar a cabo un estudio bibliométrico sobre la totalidad de las publicaciones en el área de TIC y Educación Física, con independencia de la fuente de que provengan, siempre y cuando estén accesibles en formato digital.

Los estudios bibliométricos son una rama de la cienciometría (Romaní, Huamaní, \& González-Alcaide, 2011). 
Son reconocidos por su aporte en la cuantificación de los procesos de comunicación escrita (Pritchard, 1969; Mingers \& Leydesdorff, 2015) y a partir de la aplicación de métodos estadísticos y matemáticos (Rehn \& Kronman, 2008), los que se convierten en un set de herramientas con suficiente poder metodológico para describir propiedades internas y externas de un dominio científico de conocimientos (Estabrooks, Winther, \& Derksen, 2004).

El análisis llevado a cabo en este artículo se apoya en leyes de la bibliometría, tales como productividad, dispersión, crecimiento exponencial y obsolescencia y en los indicadores bibliométricos: personal, producción, dispersión, visibilidad, colaboración y obsolescencia (Sancho, 1990).

\section{Metodología}

Se realizó un estudio descriptivo longitudinal de carácter censal de la producción científica sobre la integración de las Tecnologías de la Información y la Comunicación en la Educación Física. Se consideró la Educación Física desde su dimensión escolar formal.

\section{Fuentes de datos}

Los datos fueron recolectados desde Scopus, WOS, Latindex, Scielo, Ebsco, ERIC, Sciencedirect, Springerlink, IEEE explore, repositorios de tesis, Spacenet Patent Search, Microsoft Academic Research, ERIC, Pubmed y Google Academic. La captura de datos se realizó entre noviembre de 2017 y enero de 2018.

\section{Recolección de datos}

El proceso se dividió en cuatro etapas:

1. Se efectuó una búsqueda directa en repositorios con los términos «ICT» y «Physical Education», las que fueron ajustadas para incluir elementos taxonómicos análogos y subordinados («educational technology», «technology integration», «video technology», «blended learning», entre otros) y su representación en varios idiomas. Se utilizó el software «Publish or Perish» en su versión 6, como mecanismo auxiliar en el proceso de búsqueda por su utilidad en la producción de resultados integrados.

2. Se llevó a cabo el proceso de limpieza de información para las entradas capturadas, donde se mezclaron los resultados de diferentes fuentes y se eliminaron registros duplicados. Para la selección de registros relevantes se consideró un filtro con las siguientes condiciones: (1) Estar relacionado con el proceso educativo formal, (2) Ser publicado en una fuente indizada, (3) Tener una estructura metodológica relacionada con la comunicación de los resultados de una investigación o de experiencia innovadora en el dominio de conocimientos.

3. Se condujo una búsqueda en las referencias bibliográficas de cada uno de los artículos para incluir fuentes no encontradas en la primera etapa de búsqueda.

4. Se codificó la información obtenida como parte del proceso de análisis-síntesis. En la mayoría de los casos se recuperó la obra a texto completo. Se llevó a cabo la búsqueda de filiación de los autores, país de pertenencia, entre otros elementos. La filiación de autores fue recuperada de forma manual desde Scopus, WOS, perfiles de Google académico y Researchgate según cada caso. Se capturaron todos los metadatos significativos y se sistematizaron.

El resultado final del análisis arrojó un total de $n=521$ contribuciones, las que corresponden a todos los casos que satisfacen el filtro establecido.

\section{Tratamiento y análisis de datos}

Para la recolección de metadatos se utilizó la herramienta Zotero 5.0.x. Con ella se capturaron todos los datos disponibles de cada contribución. Se utilizó el complemento «zotero scholar citations» para determinar el número de citas de cada contribución.

Los datos obtenidos se exportaron en formato RIS, se importaron en una base de datos relacional compatible con SPSS para análisis estadístico y se utilizó Nudist Vivo 12 para análisis cualitativo. Se utilizó Vosviewer (van Eck \& Waltman, 2009) como herramienta auxiliar en el procesamiento y representación gráfica de los resultados.

\section{Resultados}

A partir del análisis de los resultados, a continuación se exponen los principales hallazgos.

\section{Principales líneas de investigación}

Se utilizó una estrategia de codificación emergente para clasificar la documentación recopilada. Se obtuvo como resultado la determinación de 70 nodos significativos relacionados a temas de investigación, de los cuales se representan y categorizan los 35 más significativos:

1. Pedagogía, con centro en la concepción de modelos, estrategias y metodologías para la enseñanza.

2. Sociología, con centro en el estudio sobre impacto social, percepciones, creencias, motivación, barreras y facilitadores.

3. Tecnología. Sobre este tema existe un incremento significativo en los últimos años sobre videojuegos activos, móviles y cuantificación personal y se ha trabajado considerablemente en el desarrollo de sistemas a la medida.

4. Comunicación, con énfasis en internet en sentido general, las redes sociales y los servicios educativos de la web 2.0.

5. Integración de TIC, con énfasis en los procesos, las barreras y facilitadores.

En la figura 1 se representan los principales temas de investigación organizados por área según color.

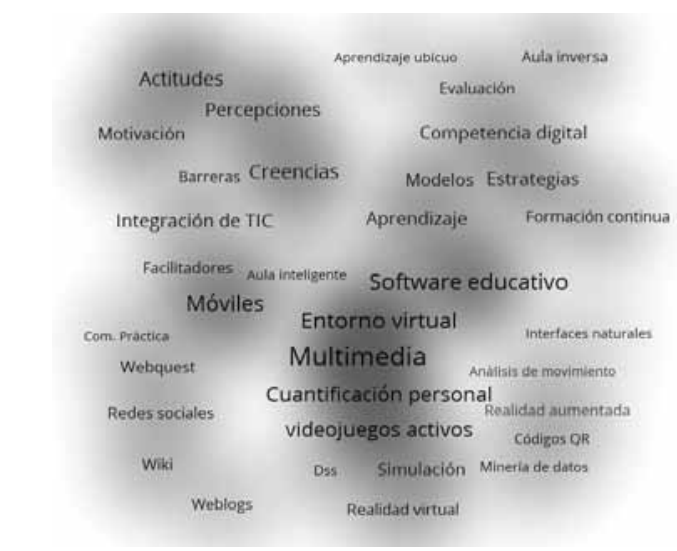

Figura.1: Principales líneas de investigación. Fuente: elaboración propia. 
Se consideran y agrupan un conjunto de propuestas que resultan relevantes desde la lógica de cuán recurrente ha sido su estudio dentro del dominio de conocimiento:

Videojuegos activos: En el análisis se constata una línea estable de trabajo en el área de videojuego activo, también llamados «exergames» o juego electrónico serio. Se asocia a socialización (Kooiman \& Sheehan, 2015), al desarrollo de la motricidad (Nyberg \& Meckbach, 2017; Vernadakis, Papastergiou, Zetou, \& Antoniou, 2015), al rendimiento cognitivo (Kooiman \& Sheehan, 2014), a la generación de estilos de vida saludable (Finco \& Zaro, 2012) y la motivación de los estudiantes por la práctica de actividades físicas (Lwin \& Malik, 2012; Nation-Grainger, 2017; Sun, 2013), entre otros. Es común encontrar referencia a las consolas que se distribuyen para el sector doméstico.

Dispositivos usables: En la literatura existe un interés creciente en el uso de dispositivos usables como medio para controlar la actividad física desde la clase de Educación Física (Goodyear, Kerner, \& Quennerstedt, 2019; Lindberg, Seo, \& Laine, 2016; Omura, Carlson, Paul, Watson, \& Fulton, 2017; Rich \& Miah, 2017). Sin embargo, todavía queda un camino por recorrer desde la perspectiva de la cultura de uso cotidiano de estos medios como forma de cuantificación personal (Geib et al., 2015), y no se encontraron referencias en cuanto a su confiabilidad como instrumentos de medición.

Sitios y servicios de la web: El uso de servicios web posee un amplio tratamiento en la literatura. Se encuentran reportes sobre el uso de WebQuest, Entornos virtuales de aprendizaje, Wikis, comunidades virtuales, redes sociales, repositorios de recursos, entre otros (López, 2013; Rodera Bermúdez, 2008; Romero, Capllonch, \& Latorre, 2005; Woods, Shimon, Karp, \& Jensen, 2004).

Dispositivos electrónicos portátiles: El uso del teléfono celular, tabletas electrónicas y asistentes personales digitales ha tenido un amplio tratamiento en la literatura (Armour, Evans, Bridge, Griffiths, \& Lucas, 2016; Crawford \& Fitzpatrick, 2015; Dimitropoulos, 2016; Hung, Shwu-Ching, \& Lin, 2017; Rosenthal \& Eliason, 2015; Yang, Maher, \& Conroy, 2015; Castro \& Gómez, 2015), más en los recientes años a partir de la aparición de entornos que facilitan la gestión e instalación de aplicaciones y la aparición de equipos con múltiples sensores que integran funciones de videocámaras, brújulas, podómetros, cronómetros, reproductores de audio y video, entre otros.

Percepción y actitud de docentes y uso de las TIC en la Educación Física: Un número importante de autores coinciden en las carencias que tienen los docentes y sobre cómo ellas influyen en la percepción sobre la utilidad de las tecnologías en la clase de Educación Física (Baert, 2011b; Gibbone, Rukavina, \& Silverman, 2010; Jackson, 2015), lo cual sin dudas condiciona la actitud que finalmente manifiestan (Bisgin, 2014).

\section{Contribuciones por país}

En el análisis de los aportes por país, se encuentra que los orígenes más productivos en el dominio de conocimientos son China, Estados Unidos y España. En la figura 2 se muestran los principales orígenes según la cantidad de autores que han publicado sobre el tema.

En la figura 3 se observa una situación similar a la mostra-

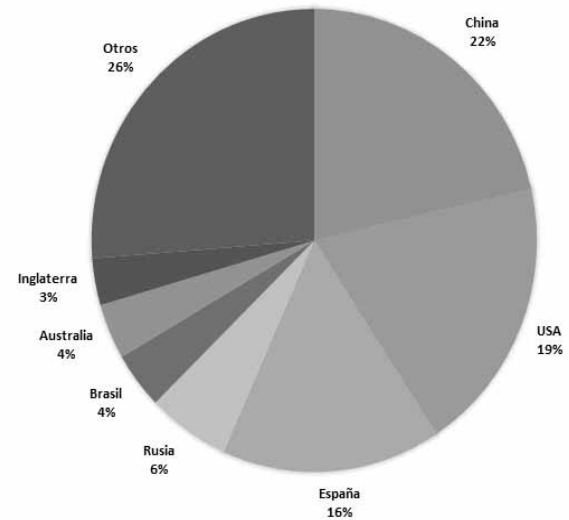

Figura.2: Distribución de autores por país. Fuente: elaboración propia.

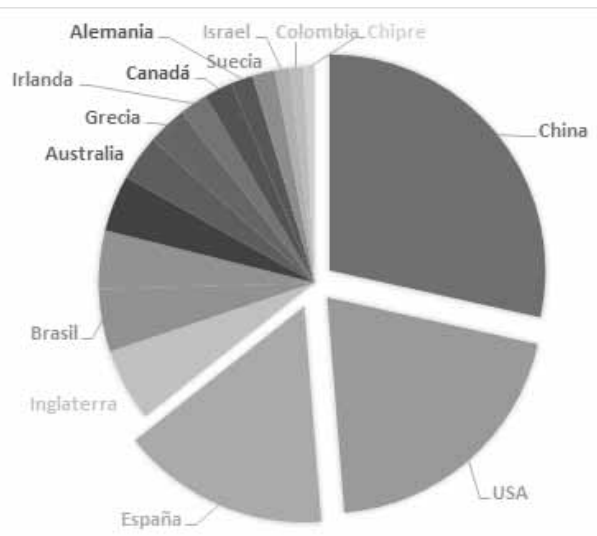

Figura.3: Contribuciones publicadas por país. Fuente: elaboración propia

da en el gráfico anterior, sin embargo, se puede notar que Inglaterra, Suecia, Irlanda y Canadá destacan por el nivel de productividad de sus autores.

En el análisis de la producción regional se encuentra que el número de contribuciones de investigadores chinos ha crecido aceleradamente en los últimos años. En un análisis más profundo se pudo constatar que un número no despreciable de esas publicaciones está relacionado con desarrollos tecnológicos publicados por investigadores que pertenecen al dominio de las ciencias de la computación, fundamentalmente en relación con la industria del software y su impacto en la clase de Educación Física. Ver figura 4.

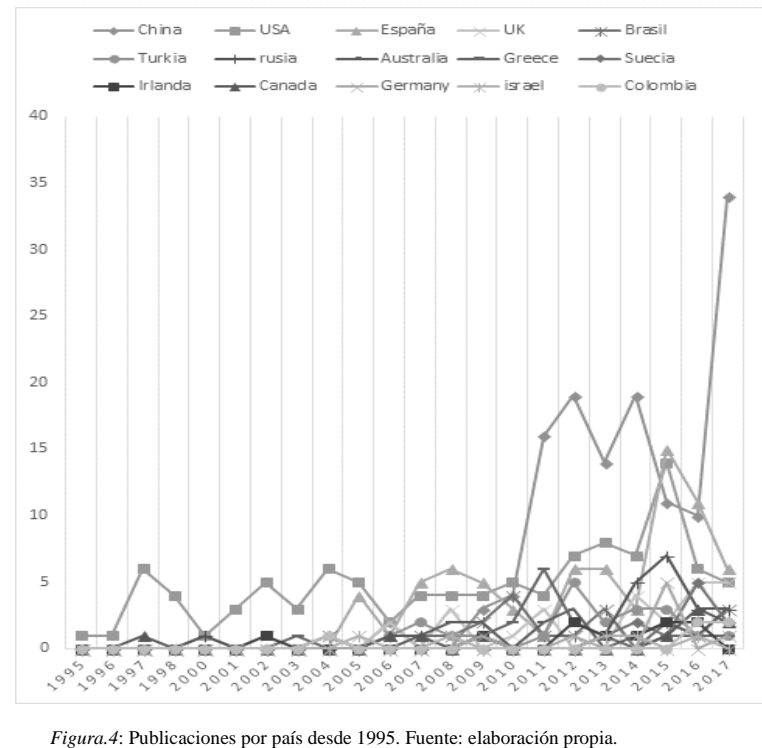




\section{Autores más influyentes}

En las obras analizadas se encuentra un total de $n=869$ autores. Se estableció un índice compuesto entre el grado de productividad en el área y el número de citas totales recibidas para representar la relevancia de los autores. Se pudo identificar que existe un amplio número de autores (ver figura 5) que se constituyen en referente en el área. La posterior revisión de la obra de los autores identificados permitió verificar la autenticidad de la selección por la madurez metodológica y la calidad de los aportes realizados por cada uno de ellos.

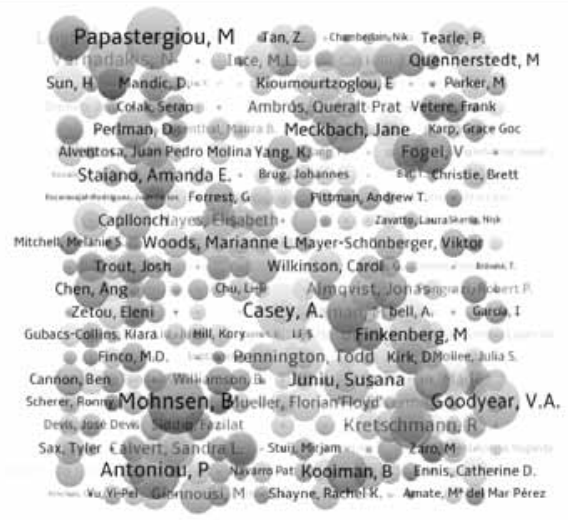

Figura.5: Relevancia de autores según productividad y citas. Fuente: elaboración propia

\section{Revistas científicas con más publicaciones en el domi- nio}

Los resultados analizados se encuentran distribuidos en 229 fuentes. De ellas, 21 revistas científicas han publicado el 34 por ciento de las publicaciones recopiladas sobre el tema (ver figura 6).

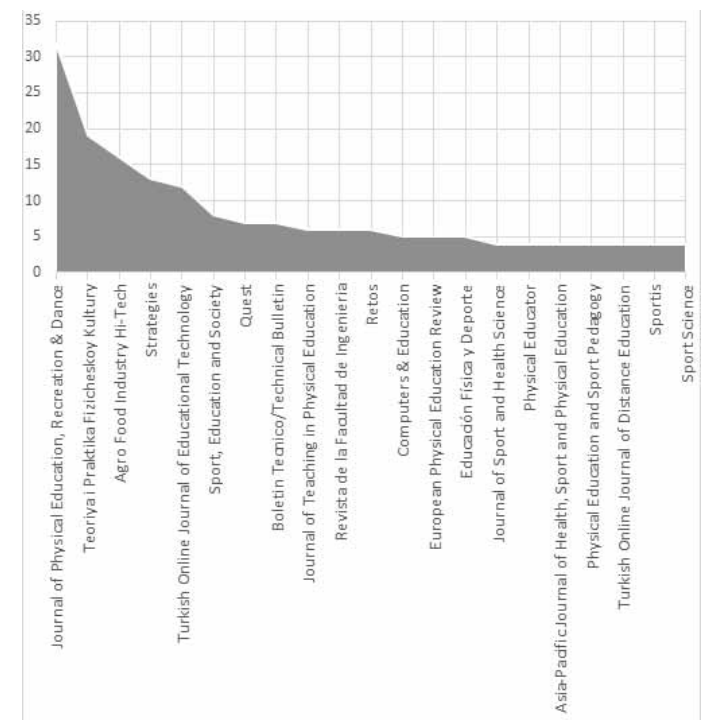

Figura.6: Fuentes con más resultados sobre TIC y Educación Física. Fuente: elaboración propia.

Uno de los primeros hallazgos que resulta interesante es que, aun cuando se ha investigado sobre el tema por más de 20 años, en el análisis de las fuentes recopiladas no se pudo identificar ninguna revista científica con algún tipo de especialización hacia el dominio de conocimiento ni conferencias científicas que tengan declaraciones explícitas sobre las TIC en la Educación Física en sus convocatorias.

En el análisis de las fuentes se encuentra una marcada tendencia a la publicación sobre el tema en fuentes naciona- les. Tal es el caso de la revista «Journal of Physical Education, Recreation \& Dance» y la revista «Strategies Journal», que han publicado fundamentalmente autores norteamericanos. La revista «Teoriya i Praktika Fizicheskoy Kultury» publica trabajos de autores rusos. Es una excepción la fuente «Agro Food Industry Hi-Tech Journal», cuyas publicaciones en el dominio corresponden a autores chinos.

\section{Madurez del dominio de conocimientos}

El número de contribuciones sobre el tema ha tenido un crecimiento estable en el periodo analizado. Según la ley de crecimiento exponencial, el crecimiento de la literatura puede ser dividido en tres etapas (de Solla, 1963). Según esta referencia, la primera etapa está determinada por las primeras publicaciones sobre el tema, la segunda está relacionada con un crecimiento exponencial a partir del interés en el dominio de conocimientos y la tercera etapa se torna lineal a partir de la reducción en la cantidad de publicaciones y una orientación más clara en la revisión y preservación del conocimiento.

En el análisis de la ventana de tiempo 1995-2017 se ha podido establecer que existe una tendencia muy clara al aumento de las publicaciones sobre TIC y Educación Física, lo que es evidencia de que actualmente el dominio se encuentra en la segunda etapa, de maduración. En este caso se considera por los autores la primera fase entre los años 1995 y 2003(Ver figura 7).

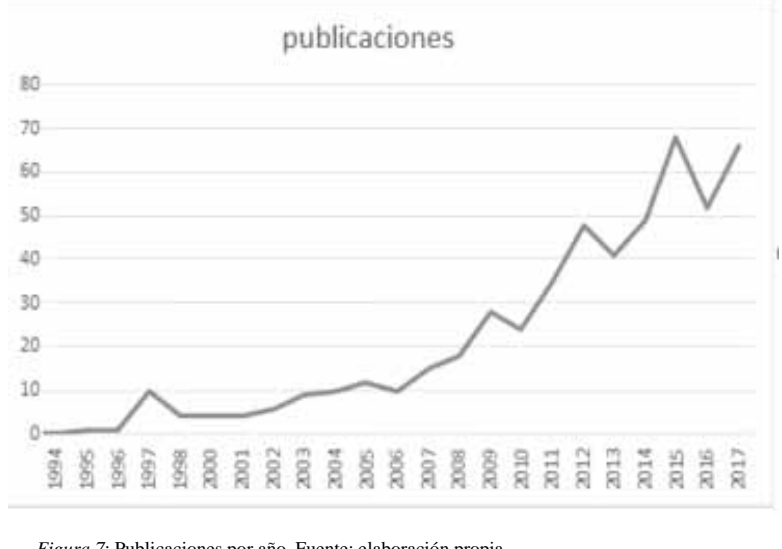

La necesidad actual de que se investigue el impacto de la tecnología sobre la transformación didáctica de la Educación Física y la disponibilidad cada vez más alta de equipamiento portable y vestible en las aulas, llevará inevitablemente a que el dominio siga creciendo. Igualmente constituye una línea de trabajo necesaria los múltiples llamados que se encuentran en la literatura reciente a que se establezca un análisis mucho más centrado en la dimensión pedagógica de la Integración de las TIC.

Como complemento al análisis, se aplicó el índice Burtonkebler (Burton \& Kebler, 1960), para encontrar el grado de obsolescencia en la literatura donde se encontró que la vida de las publicaciones tiene un semiperiodo de seis años con una desviación de 7.6 y en la aplicación del índice de Price (Gómez, 2005; Sancho, 1990), se obtiene que hay un 46.2 por ciento de la literatura citada al menos una vez en los siguientes cinco años de haber sido publicada.

Aunque ambos resultados podrían dar fe de la velocidad 
con que cambia la tecnología, más que de la velocidad con que se renueva el conocimiento en el área, en cualquiera de los casos se refiere a un dominio en que los resultados de investigación se consideran obsoletos con relativa prontitud.

En el análisis de inmediatez se pudo constatar que existe un índice de inmediatez bajo, donde sólo un número limitado de artículos en el dominio es referenciado en el primer año de publicación, aun cuando es cada vez más común que las publicaciones queden disponibles hasta un año antes de su fecha de publicación formal. Ello puede estar asociado al tiempo que toma publicar una obra a los autores noveles en comparación con los autores ya reconocidos y en relación con el alto nivel de transitividad del dominio.

En el análisis de la relación entre publicaciones y autores se puede constatar que existe un número sumamente alto $\mathrm{n}=734$ de autores que publica sólo una obra y que solo $\mathrm{n}=134$ autores han publicado al menos dos contribuciones en el área. Aunque este es un fenómeno natural y no significa que se dejó de investigar en el tema, podría plantear alertas sobre la limitada magnitud de la comunidad de autores que trabajan de forma estable en el dominio de conocimientos.

\section{Colaboración académica}

El dominio muestra un alto grado de colaboración académica nacional según se ha podido constatar en el análisis de la literatura. Del total de artículos recopilados, el 8.8 por ciento ha sido firmado por cinco o más autores, con un máximo de 10 autores y una media de 2.31 autores por contribución.

Aunque un número tan alto de coautoría sugiere altos niveles de colaboración, en la lectura de las contribuciones con alto número de coautoría no queda siempre clara la referencia a marcos de colaboración que justifiquen dicha coproducción. Tampoco existe correspondencia entre la cantidad de autores que firman la obra y la cantidad de veces que es referenciada la obra, por lo que no se atribuye un impacto positivo en este sentido. Por otra parte, el 36 por ciento de las obras analizadas fueron firmadas por un solo autor,

Un punto de interés en el análisis lo constituye la coautoría internacional, la cual puede ser considerada una vía para identificar redes de colaboración. (Ver figura 8). Si la coautoría puede explicar la colaboración internacional en el dominio, queda claro entonces que todavía estamos en un contexto en el que faltan iniciativas de colaboración internacional en cuanto a la integración de las TIC en la Educación Física.

El análisis expone una baja colaboración entre 1997 y

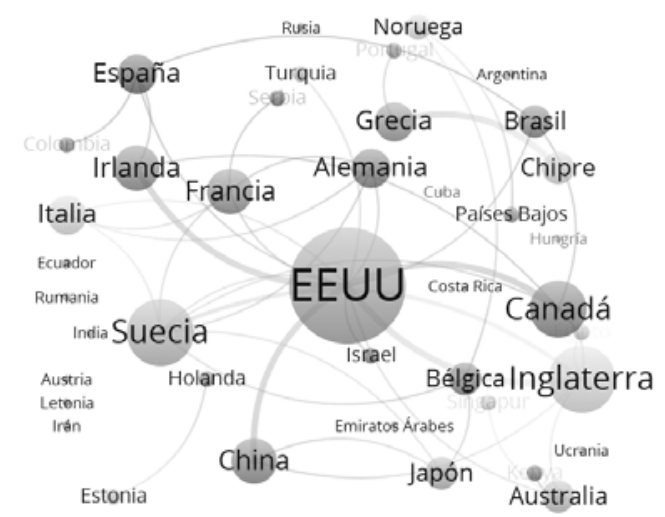

Figura. 8: Colaboración internacional en el dominio. Fuente: elaboración propia.
2010, donde solo 10 publicaciones responden a colaboración internacional. Desde 2011 en adelante se constata un aumento, aunque discreto, en esta dirección.

En todo el periodo estudiado se observa que investigadores de EEUU, Inglaterra, Suecia, Canadá, Francia y Alemania han mantenido colaboración en redes internacionales, mientras que autores de otros países tienen colaboraciones bidireccionales o adolecen de ellas.

\section{Análisis de citas}

En el análisis de citas para las contribuciones recopiladas se pudo valorar el bajo número de referencias para las publicaciones en el dominio. El 51.65 por ciento de las contribuciones nunca ha sido referenciado desde una fuente indizada, según consta en el Google Académico y en el análisis de co-citación para los documentos recuperados.

El 15.6 por ciento de las publicaciones analizadas ha sido referenciado entre una y tres veces en todo el ciclo de vida. El 12 por ciento tiene entre cuatro y diez citas y solo el 19.5 por ciento ha sido referenciado más de diez veces.

Es significativo el hecho de que la mayoría de las contribuciones citan, fundamentalmente, publicaciones relacionadas con la psicología, tecnología, filosofía, contribuciones generales relacionadas con educación y la tecnología educativa y que en muchos casos se ignoran trabajos relevantes en el dominio de conocimiento.

El análisis de co-citación arroja un número notable de auto citas o de citas a autores de la misma institución, lo que es interesante como medio para el debate local y la posible conformación de escuelas de pensamiento.

Los autores con obras más citadas corresponden a EEUU, Grecia, Australia, Inglaterra, España y Turquía. El análisis de citas por documento arroja, como resultados más relevantes, un total de 54.57 citas por documento como promedio para autores de EEUU, 42 para autores de Holanda, 18 para autores de Australia y 10.8 citas por publicación para autores de Inglaterra.

\section{Conclusiones}

A partir del estudio bibliométrico de la literatura escrita publicada entre 1995 y 2017, recopilada desde los principales repositorios científicos, sin distinción según niveles de indización o rigor científico, y con el objetivo de describir el dominio de conocimientos asociado a las TIC y la Educación Física, se llega a las siguientes conclusiones:

Los principales temas investigados en los últimos años han estado relacionados a la pedagogía, la tecnología, la sociología, la tecnología, la comunicación y la gestión de la integración de las TIC. Destaca entre ellos, en cuanto a frecuencia, la investigación sobre videojuegos activos, dispositivos usables, sitios y servicios web, dispositivos portátiles y percepción y actitud de los docentes hacia el uso de las TIC.

Las publicaciones sobre el tema se polarizan fundamentalmente en Estados Unidos, China, España, Inglaterra, con una presencia algo menor en otros países de Europa y una baja presencia en América Latina.

El número de publicaciones en el dominio crece de forma estable, por lo que si se toma en cuenta la masificación que 
ha tenido el acceso a la tecnología en los últimos años, podemos concluir que estamos ante un dominio de conocimientos que todavía está en crecimiento.

El dominio se caracteriza por un alto nivel de transición, sobre la base del bajo número de autores que publica más de una obra relacionada con TIC y Educación Física.

En el análisis de filiación de los autores de cada trabajo se percibe un alto nivel de colaboración nacional y un bajo nivel de colaboración académica internacional en el dominio de conocimientos.

Las publicaciones en el dominio son poco citadas (el 51.65\% nunca fue citado), con excepción de publicaciones de un grupo reducido de autores. Igualmente se encuentra que, como promedio, la literatura se hace obsoleta a los seis años de publicada.

\section{Referencias}

Araújo, J. G. E., Batista, C., \& Moura, D. L. (2017). Exergames in physical education: A systematic review. Movimento, 23(2), 529-542.

Armour, K. M., Evans, G., Bridge, M., Griffiths, M., \& Lucas, S. (2016). Gareth: The beauty of the ipad for revolutionising learning in physical education. In Digit. Technol. and Learn. in Phys. Educ.: Pedagogical Cases (pp. 213-230). Taylor and Francis Inc. DOI: 10.4324/ 9781315670164

Baert, H. (2011a). The integration of technology within physical education teacher education: Perceptions of the faculty. University of Arkansas.

Baert, H. (2011b). The integration of technology within physical education teacher education: Perceptions of the faculty. University of Arkansas.

Bauer, J., \& Kenton, J. (2005). Toward technology integration in the schools: Why it isn't happening. Journal of Technology and Teacher Education, 13(4), 519.

Bisgin, H. (2014). Analyzing the Attitudes of Physical Education and Sport Teachers towards Technology. Anthropologist, 18(3), 761-764.

Burton, R. E., \& Kebler, R. W. (1960). The «half-life» of some scientific and technical literatures. Journal of the Association for Information Science and Technology, 11(1), 18-22.

Campos, C. M., \& del Castillo Fernández, H. (2016). The benefits of active video games for educational and physical activity approaches: A systematic review. Journal of New Approaches in Educational Research, 5(2), 115.

Castro L., N., \& Gómez G., I. (2016). Incorporación de los códigos QR en la Educación Física en Secundaria. Retos: Nuevas tendencias en Educación Física, Deportes y Recreación, 29, 114-119.

Crawford, S., \& Fitzpatrick, P. (2015). Use of mobile digital technology and iPod touches in physical education. In Handb. of Mob. Teach. and Learn. (pp. 499-508). Springer Berlin Heidelberg. DOI: 10.1007/978-3-642-54146-9_72

de Solla Price, D. J. (1963). Little science, big science. Columbia University Press New York.

Dimitropoulos, A. (2016). Fitness revisited: Mobile learning in physical education. In Revolutionizing K-12 Blended Learn. through the i2Flex Classr. Model (pp. 320-333).

\section{IGIGlobal. DOI:10.4018/978-1-5225-0267-8.ch020}

Estabrooks, C. A., Winther, C., \& Derksen, L. (2004). Mapping the field: a bibliometric analysis of the research utilization literature in nursing. Nursing Research, 53(5), 293-303.

Finco, M. D., \& Zaro, M. A. (2012). Developing a healthy lifestyle through the use of exergames in physical education classes. In Proc. IADIS Int. Conf. ICT, Soc. Hum. Beings, Proc. IADIS Int. Conf. e-commerce 2012. 156-158.

Geib, R. W., Swink, P. J., Vorel, A. J., Shepard, C. S., Gurovich, A. N., \& Waite, G. N. (2015). The bioengineering of changing lifestyle and wearable technology: A mini review. In Annu. Rocky Mt. Bioeng. Symp. Int. ISA Biomed. Sci. Instrum. Symp. (pp. 71-78). International Society of Automation (ISA).

Gibbone, A., Rukavina, P., \& Silverman, S. (2010). Technology integration in secondary physical education: teachers' attitudes and practice. Journal of Educational Technology Development and Exchange (JETDE), 3(1), 3.

Gómez, C. F. R.-C. (2005). Indicadores bibliométricos: origen, aplicación, contradicción y nuevas propuestas. MedUNAB, 8(1).

Goodyear, V.A., Kerner, C., \& Quennerstedt, M. (2019). Young people's uses of wearable healthy lifestyle technologies; surveillance, self-surveillance and resistance. Sport, education and society, 24(3), 212-225.

Hung, H.-C., Shwu-Ching Young, S., \& Lin, K.-C. (2017). Exploring the effects of integrating the iPad to improve students' motivation and badminton skills: a WISER model for physical education. Article in Press. DOI: 10.1080/1475939X.2017.1384756

Jackson, J. M. (2015). An examination of the perceptions of online physical education: How fit is online PE? Bowling Green State University.

Kooiman, B. J., \& Sheehan, D. P. (2015). The efficacy of exergames for social relatedness in online physical education. Cogent Education, 2(1), 1045808.

Kretschmann, R. (2015). Physical education teachers' subjective theories about integrating information and communication technology (ICT) into physical education. Turkish Online Journal of Educational Technology, 14(1), 68-96.

Lindberg, R., Seo, J., \& Laine, T. H. (2016). Enhancing Physical Education with Exergames and Wearable Technology. IEEE Transactions on Learning Technologies, 9(4), 328341. DOI: $10.1109 /$ TLT.2016.2556671

López, M. G. (2013). La webquest en la enseñanza del balonmano. Espiral. Cuadernos Del Profesorado., 6(11), 28-35.

Lwin, M. O., \& Malik, S. (2012). The efficacy of exergamesincorporated physical education lessons in influencing drivers of physical activity: A comparison of children and pre-adolescents. Psychology of Sport and Exercise, 13(6), 756-760. DOI: 10.1016/j.psychsport.2012.04.013

Macdonald, J. C. (2015). A Review Studying Wearable Technology and Augmented Reality as it may Apply to Teaching and Learning (Magister). Edinburgh.

Mingers, J., \& Leydesdorff, L. (2015). A review of theory and practice in scientometrics. European journal of 
operational research, 246(1), 1-19.

van Eck, N., \& Waltman, L. (2009). Software survey: VOSviewer, a computer program for bibliometric mapping. Scientometrics, 84(2), 523-538.

Nation-Grainger, S. (2017). 'It's just PE' till 'It felt like a computer game': using technology to improve motivation in physical education. Research Papers in Education, 32(4), 463-480. DOI: 10.1080/02671522.2017.1319590

Nyberg, G., \& Meckbach, J. (2017). Exergames ‘as a teacher of movement education: exploring knowing in moving when playing dance games in physical education. Physical Education and Sport Pedagogy, 22(1), 1-14.

Omura, J. D., Carlson, S.A., Paul, P., Watson, K. B., \& Fulton, J. E. (2017). National physical activity surveillance: Users of wearable activity monitors as a potential data source. Preventive Medicine Reports, 5, 124-126.

Papastergiou, M. (2009). Exploring the potential of computer and video games for health and physical education: A literature review. Computers \& Education, 53(3), 603622.

Pritchard, A. (1969). Statistical bibliography or bibliometrics. Journal of Documentation, 25(4), 348-349.

Quijada, M. R. (2015). Tecnologías de la Información y la Comunicación (TIC) en Educación Física. Una revisión teórica. Sportis: Revista Técnico-Científica Del Deporte Escolar, Educación Física y Psicomotricidad, 1(1), 75-86.

Rehn, C., \& Kronman, U. (2008). Bibliometric handbook for Karolinska Institutet. Huddinge: Karolinska Institutet.

Rich, E., \& Miah, A. (2017). Mobile, wearable and ingestible health technologies: towards a critical research agenda. Health Sociology Review, 26(1), 84-97.

Bermúdez, A. M. R. (2008). Catalogación y valoración de las webquests desde el área de educación física y el tratamiento de temáticas transversale. Edutec: Revista elec- trónica de tecnología educativa, (27), 4.

Romaní, F., Huamaní, C., \& González-Alcaide, G. (2011). Estudios bibliométricos como línea de investigación en las ciencias biomédicas: una aproximación para el pregrado, 11.

Romero Granados, S., Capllonch Bujosa, M., \& Latorre Romero, Á. (2005). Educación Física a través del ordenador. Las WebQuest: nuevos recursos para el alumnado. Comunicación y Pedagogía. Nuevas Tecnologías y Recursos Didácticos, 206, 58-62.

Rosenthal, M. B., \& Eliason, S. K. (2015). «I Have an iPad. Now What?» Using Mobile Devices in University Physical Education Programs. Journal of Physical Education, Recreation \& Dance, 86, 34-39. DOI:10.1080/ 07303084.2015.1053636

Sancho, R. (1990). Indicadores bibliométricos utilizados en la evaluación de la ciencia y la tecnología. Revisión bibliográfica. Revista Española de Documentación Científica, 13(3), 842.

Sun, H. (2013). Impact of exergames on physical activity and motivation in elementary school students: A follow-up study. Journal of Sport and Health Science, 2(3), 138145.DOI: 10.1016/j.jshs.2013.02.003

Vernadakis, N., Papastergiou, M., Zetou, E., \& Antoniou, P. (2015). The impact of an exergame-based intervention on children's fundamental motor skills. Computers \& Education, 83, 90-102. DOI: 10.1016/ j.compedu.2015.01.001

Woods, M. L., Shimon, J. M., Karp, G. G., \& Jensen, K. (2004). Using webquests to create online learning opportunities in physical education. Journal of Physical Education, Recreation \& Dance, 75(8), 41-46.

Yang, C.-H., Maher, J. P., \& Conroy, D. E. (2015). Implementation of behavior change techniques in mobile applications for physical activity. American Journal of Preventive Medicine, 48(4), 452-455.

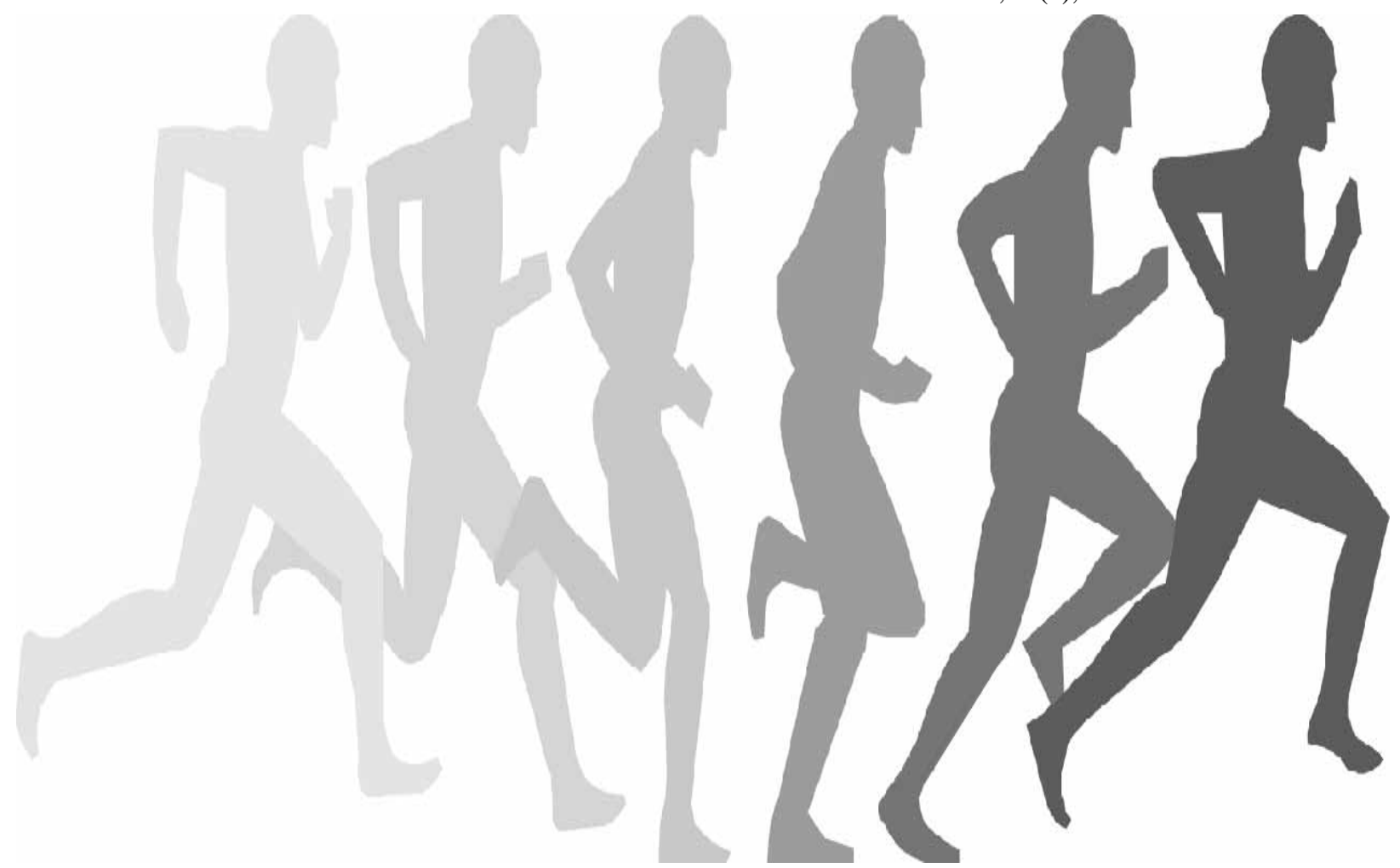

